\title{
Entropy AHP and its comparison with conventional AHP's
}

\author{
Masaaki Shinohara \\ Department of Mathematical Engineering, \\ College of Industrial Technology, Nihon University \\ 1-2-1 Izumi-cho, Narashino, Chiba 275-8575, Japan
}

\begin{abstract}
A new method of estimating the weight vector in AHP is proposed. Since this method assumes an entropy optimization model as the background model for AHP's pairwise comparison, the AHP with this weight vector estimation is called entropy-based AHP or entropy AHP. An interpretation for this entropy-based background model is presented and on the basis of this entropy-based background model, a method of estimating the weight vector is presented. Finally, estimated results of the weight vector by this entropy AHP are compared with those by conventional geometric-mean AHP and eigenvector AHP.
\end{abstract}

\section{Introduction}

A new method of estimating the weight vector $w$ in AHP is proposed. Since this method assumes an entropy optimization model as the background model for AHP's pairwise comparison, the AHP with this weight vector estimation is called entropy-based AHP or entropy AHP. In Sec. 2, a background model with entropy distance minimization is proposed for the pairwise comparison. In Sec. 3, an interpretation for this entropy-based background model is presented. In Sec. 4, on the basis of this entropy-based background model, a method of estimating the weight vector $w$ is presented. In Sec. 5 . estimated results of the weight vector $w$ by this entropy AHP are compared with those by conventional geometric-mean AHP and eigenvector AHP.

\section{Background model with entropy distance minimization for AHP's pairwise comparison}

Consider an entropy distance minimization problem EDMP ((1) (4)).

[EDMP]

$$
\begin{aligned}
& \text { Objective function : } D(X, Y)=\sum_{i=1}^{n} \sum_{i=1}^{n} x_{i j}\left(\ln \left(x_{i j} / y_{i j}\right)-1\right) \\
& \text { Decision variable: } X=\left\{x_{i j}\right\}
\end{aligned}
$$




$$
\begin{aligned}
& \text { Given variable: } Y=\left\{y_{i j}\right\}, \mathbf{p}=\left\{p_{k}\right\} \\
& \text { Constraint: } \sum_{j=1}^{n} x_{k j}-\sum_{i=1}^{n} x_{i k}=p_{k}(k=1, \ldots n)
\end{aligned}
$$

The optimality condition for EDMP is derived with the use of Lagragean function $L(X, \lambda)$.

Optimality condition:

$$
\begin{gathered}
\frac{\partial L}{\partial x_{i j}}=0 \quad(i=1, \ldots, n \text { and } j=1, \ldots, n) \\
L(X, \lambda)=D(X, Y)+\sum_{k=1}^{n} \lambda_{k}\left(\sum_{j=1}^{n} x_{k j}-\sum_{i=1}^{n} x_{i k}-p_{k}\right)
\end{gathered}
$$

The optimality condition (5) is evaluated as below.

Therefore, $x_{i j}$ is expressed as below.

$$
\frac{\partial L}{\partial x_{i j}}=\ln \left(x_{i j} / y_{i j}\right)+1+\lambda_{i}-\lambda_{j}-1=0
$$

Let $w_{i}=e^{-\lambda_{i}}$, then Eq. (9) is obtained.

$$
x_{i j}=y_{i j} \exp \left(-\lambda_{i}+\lambda_{j}\right)
$$

$$
x_{i j}=y_{i j} \frac{w_{i}}{w_{i}}
$$

Here, let $y_{i j}=1$ for all $(i, j)$ pairs, then Eq. (10) is obtained finally.

$$
x_{i j}=\frac{w_{i}}{w_{i}} \quad(i=1, \ldots n \quad \text { and } j=1, \ldots, n)
$$

\section{Interpretation for entropy-based background model}

(3.1) Decision variable $X=\left\{x_{i j}\right\}$ is the true unknown pairwise comparison matrix, whose $(i, j)$ th element is the value of item $i$ evaluated by item $j$.

(3.2) Given variable $Y=\left\{y_{i j}\right\}$ is a reference pairwise comparison matrix.

(3.3) In deciding $X$, we are trying to minimize an information theoretic distance (or an entropy distance) from $X$ to $Y$ under the constraint (4).

(3.4) Constraint (4) is a conservation law for value flows. That is, the first term of left-hand side of Eq.(4), $\sum_{j} x_{k j}$, is the total sum of input value flows of item $k$, the second term of left-hand side of Eq.(4), $\sum_{i} x_{i k}$, is the total sum of output value flows of item $k$, and therefore, the right-hand side of Eq.(4), $p_{k}$, is (a kind of) value of item $k$ (Fig.I).

The constraint (4) insists that the sum of each score of item $k$ evaluated by all items (including item $k$ ) minus the sum of each score of all items evaluated by item $k$ should equal to some value of item $k, p_{k}$. (3.5) Since it is easily shown that $\mathbf{p}=\left\{p_{k}\right\}$ satisfies Eq.(11), we call this value vector the zero-sum 
value vector. The proposed entropy AHP method transforms a zero-sum value vector $\mathbf{p}$ to a constant-sum nonnegative weight vector $w$.

$$
\sum_{k=1}^{n} p_{k}=0
$$

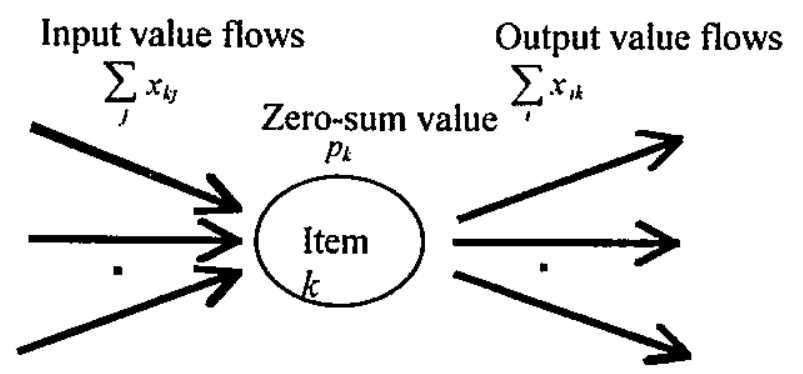

Fig. 1 Mechanism of determining zero-sum value $p_{k}$

(3.6) The $(i, j)$ th element of pairwise comparison matrix $X=\left\{x_{i j}\right\}$ which minimizes $\mathrm{D}(\mathrm{X}, \mathrm{Y})$ with a uniform reference matrix $Y\left(y_{i j}=1\right.$ for all $(i, j)$ pairs) and a zero-sum value vector $\mathbf{p}=\left\{p_{k}\right\}$ given, has a solution of the form " $x_{i j}=w_{i} / w_{j}$ "

(3.7) The uniformity of reference matrix $Y$ indicates that there is no information about the pairwise comparison matrix to be compared to.

\section{Weight vector estimation}

Given the uniform reference matrix $\mathrm{Y}$ and zero-sum value vector $\mathrm{p}$, the pairwise comparison matrix $\mathrm{X}$ (or the weight vector $w$ ) can be determined by solving following set of nonlinear equations NLE ((12)(14)), the optimality condition for EDMP.

[NLE]

$$
\begin{gathered}
x_{i j}=\frac{w_{i}}{w_{i}} \quad(i=1, \ldots, n \quad \text { and } j=1, \ldots, n) \\
\sum_{j} x_{k j}-\sum_{1} x_{i k}=p_{k} \quad(k=1, \ldots, n) \\
\sum_{k} w_{k}=1
\end{gathered}
$$

\section{Numerical experiment}

Proposed entropy AHP is compared with geometric-mean AHP and eigenvector AHP for two classes of randomly generated pairwise comparison matrices of size $n=4$.

[Numerical example 1]

Upper elements of a pairwise comparison matrix $\mathrm{A}\left(a_{i j}\right.$ 's with $\left.i<j\right)$ are determined by Eq.(15).

$$
a_{i j} \leftarrow \text { integer randomly chosen from } 1 \text { to } 10
$$


Lower elements ( $a_{i j}$ 's with $i>j$ ) are determined by Eq.(i6).

$$
a_{i j} \leftarrow 1 / a_{j i}
$$

Diagonal elements ( $a_{i i}$ 's) are all l's.

Using the pairwise comparison matrix $A=\left\{a_{i j}\right\}$, the zero-sum value vector $\mathbf{p}=\left\{p_{k}\right\}$ is given by Eq.(17).

$$
p_{k}=\sum_{j} a_{k j}-\sum_{i} a_{i k}
$$

The standard deviation $\mathrm{s}_{\mathrm{w}}$ for a constant-sum nonnegative weight vector $\mathrm{w}$ is calculated by Eq.(18).

$$
\begin{aligned}
& s_{w}=\sqrt{S /(n-1)} \\
& S=\sum_{i=1}^{n}\left(w_{i}-\frac{1}{n}\right)^{2}
\end{aligned}
$$

The square-root distance $d_{k l}$ between $\mathbf{w}^{k}$ and $\mathbf{w}^{\prime}$ is calculated by Eq.(20).

$$
d_{k l}=\sqrt{\sum_{i=1}^{n}\left(w_{i}^{k}-w_{i}^{l}\right)^{2}}
$$

Figure 2 shows the standard deviation $s_{w}$ for the three AHP methods and three square-root distances, $d_{12}$, $d_{23}$ and $d_{13}$, averaged over 100 randomly generated $A$ 's.

Here, suffix 1 stands for geometric-mean AHP, 2 for eigenvector AHP and 3 for entropy AHP. Table 1 shows the detail data for one sample out of the 100 randomly generated A's.

[Numerical example 2]

True weight vector $\mathbf{w}^{*}$ is assumed as below.

$$
\mathbf{w}^{*}=(0.4,0.3,0.2,0.1)
$$

Using this true weight vector, upper elements of a pairwise comparison matrix $A$ are determined by Eq.(22).

$$
a_{i j} \leftarrow w_{i}^{*} / w_{j}^{*}
$$

Then, real random number uniformly distributed over $(-0.1,0.1)$ is added to $a_{i j}(i<j)$.

Lower elements and diagonal elements are determined by Equs.(23) and (24).

$$
\begin{array}{cc}
a_{i j} \leftarrow 1 / a_{j i} & (i>j) \\
a_{i j}=1 & (i=j)
\end{array}
$$

Then, the zero-sum value vector $\mathbf{p}=\left\{p_{k}\right\}$ is calculated by Eq.(17).

Figure 3 shows the standard deviation $s_{w}$ for the three AHP methods and the three square-root distances, $d_{12}, d_{23}$, and $d_{13}$, averaged over 100 randomly generated $A$ 's. 
From the numerical experiment results of Example 1 and Example 2, followings are observed.

1. The pattern of weight vectors obtained by the entropy AHP is not similar to those by the geometricmean AHP and eigenvector AHP, because the square-root distances $d_{23}$ and $d_{13}$ are about 2 to 10 times bigger than the square-root distance $d_{12}$.

2. The same tendency that $d_{12}<d_{23}$ and $d_{12}<d_{13}$ can be observed also with other distance metrics, such as absolute value $\left(\mathrm{L}_{1}\right.$-metric) distance and Kullback-Leibler information theoretic distance.

3. The distribution of weight values within a weight vector can be evaluated by the standard deviation $\mathrm{s}_{\mathrm{w}}$ defined by Eq.(18). Although $\mathrm{s}_{\mathrm{w}}$ values can be different among the three AHP methods from one sample to another, the $s_{w}$ values averaged over 100 samples are nearly the same, or no significant difference in $s_{w}$ is observed among the three AHP methods.

\section{Conclusion}

A new method of estimating the weight vector $w$, which is based on an entropy optimization background model, or minimizing Kullback-Leibler information theoretic distance (cross-entropy), is proposed.

Proposed entropy AHP method requires as input data a zero-sum value vector $\mathbf{p}=\left\{p_{k}\right\}$ with a property that $\sum p_{k}=0$ and produces a constant-sum nonnegative weight vector $\mathbf{w}=\left\{w_{k}\right\}$ with a property that $\sum w_{k}=1$ and $w_{k} \geq 0$. Therefore, in the framework of proposed entropy AHP, AHP functions as a transformation from $\mathrm{p}$ to $w$. Although pairwise comparison matrix $A$ is used to estimate a zero-sum value vector $p$ in our numerical examples, we insist that measurement of zero-sum value vector $p$ alone is essential for proposed entropy AHP.

\section{Reference}

[1] Thomas L. Saaty: The Analytic Network Process (RWS Publications, 1996)

\section{Acknowledgement}

The author expresses his gratitude to Professor Iwaro Takahashi of Nihon University for his useful suggestions and sincere guidance. 

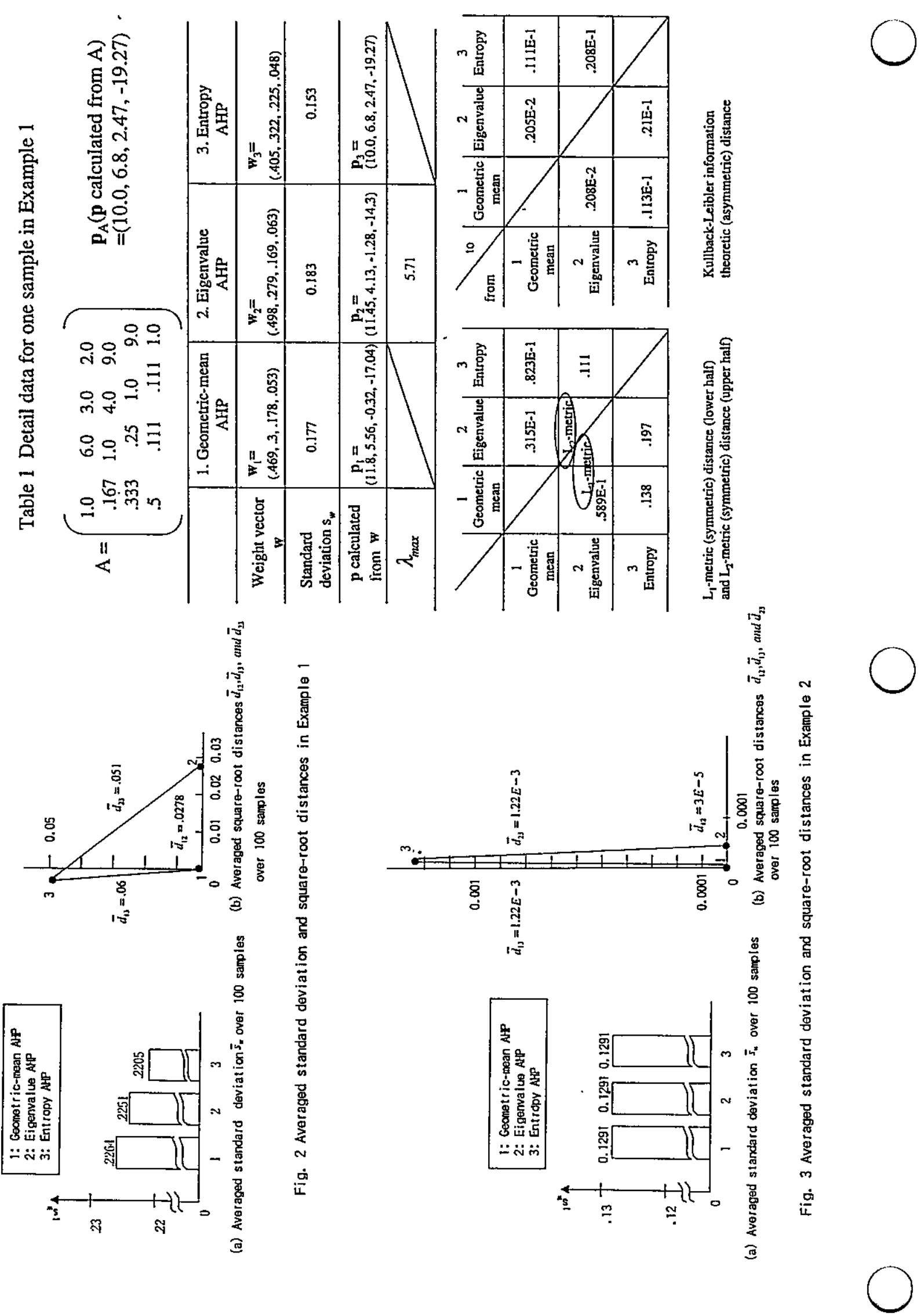